\title{
Coronary circulation in acute myocardial infarction $^{1}$
}

\author{
Thomas N. James \\ From the Cardiovascular Research and Training Center, University of Alabama School of \\ Medicine, Birmingham, Alabama 35233, U.S.A.
}

$A$ brief review is presented on the coronary circulation in acute myocardial infarction. The format of presentation is general anatomy of the human coronary arteries, the blood supply of certain special regions of the heart, and a consideration of how ischaemic malfunction in these regions leads to some of the clinical problems encountered in the management of the patient with acute myocardial infarction.

Certain recent observations have raised important questions concerning the role of the coronary circulation in acute myocardial infarction. For example, it has been reported that thrombotic occlusion may develop after rather than before the infarction (Spain and Bradess, 1960; Roberts and Buja, 1970), and even that myocardial infarction may occur in the absence of coronary disease (Kemp, Elliott, and Gorlin, 1967; Eliot and Bratt, 1969). While the questions raised by such observations will undoubtedly lead to a better understanding of the pathophysiology of acute myocardial infarction, we should not lose sight of the fact that the overwhelming majority of clinical examples of this disease are found in patients with grossly pathologic coronary arteries. Whether the arteries are completely occluded or only greatly narrowed, and whether the process is recent or ancient, are questions which must not distract us from the most plausible interpretation: that myocardial infarction and coronary disease are causally related and that the rare exceptions simply prove the rule.

The clinical course of patients with myocardial infarction is determined to a significant extent by the anatomical location of the infarct (James, 1968). One clear example is the close relationship between site of infarct and the pathogenesis of arrhythmias and heart block, which in turn have much to do with whether the heart continues to pump normally or not. The anatomical sites of pacemaking and conduction have a specific blood

1 This work was supported in part by grants from the U.S. Public Health Service. supply, as have other critically important structures such as the papillary muscles and the interventricular septum. The purpose of this review is to describe briefly the general anatomy of the coronary arteries (James, I96I), then the blood supply of special regions in the human heart, and finally to consider how ischaemic malfunction in those regions of the heart contributes to the problems encountered in the clinical management of the patient with acute myocardial infarction.

General anatomy of the coronary arteries

The main left coronary artery is usually one or two centimetres long and has normally no branches proximal to its 'bifurcation'. This initial division into two major trunks most often includes a third or even fourth major trunk at the same point (Fig. I). It is important to appreciate that large additional branches (known as diagonal branches) are normally present at this site, since their occlusion may produce myocardial infarction but can be overlooked in a coronary arteriogram.

The left anterior descending coronary artery (Fig. 2) is virtually a continuation of the main left coronary trunk, coursing down the anterior interventricular sulcus to the apex cordis, where instead of terminating it curves up into the posterior interventricular sulcus and ascends a distance of 2 to 5 centimetres. It supplies most of the interventricular septum (discussed later), a small adjacent margin of the free wall of the right ventricle, and a large portion of the adjacent free wall 
of the left ventricle. Its branches originate at acute angles.

The left circumflex coronary artery originates from the left coronary trunk at an angle of $90^{\circ}$ or more (Fig. 3) and passes beneath the left atrial appendage into the atrioventricular (AV) sulcus, where it continues to the obtuse margin of the heart. There it usually terminates by descending as the left marginal artery, sending a variable number of branches to the diaphragmatic surface of the left ventricle. Its branches, like those of the anterior descending, arise at acute angles.

The right coronary artery is a counterpart to the left circumflex branch, coursing in the opposite AV sulcus. It lies more deeply placed in the fat of the AV sulcus and its branches originate at right angles (Fig. 4). In the right coronary sinus of the aorta there is in 50 per cent of human hearts a small separate ostium giving origin to the conus artery (Schlesinger, Zoll, and Wessler, 1949). The conus artery in such cases cannot be visualized with selective right coronary arteriograms, although it is the first major branch of the right coronary in the remaining 50 per cent of human hearts. The right coronary terminates on the diaphragmatic surface of the heart (in $90 \%$ of human hearts) with two or more branches descending in or parallel to the interventricular sulcus toward the apex cordis (Fig. 5). These branches seldom extend as far as the apex, which is supplied almost entirely by branches of the left anterior descending coronary artery.

Interarterial anastomoses are abundantly present in the normal human heart, frequently being several hundred microns in diameter. They progressively enlarge and become twisted in shape in hearts with coronary occlusive disease (Fig. 6). Regions containing particularly important anastomoses are the anterior free wall of the right ventricle, the interventricular septum, the atria, and the

FIG. 2 The typical course of the left anterior descending artery (two arrows) is illustrated here. White plastic is in the right cardiac chambers and the veins, but the left chambers are uncast.

FIG. 3 The normal left circumflex coronary artery (straight arrow) is shown coursing to the obtuse margin of the left ventricle, where it terminates as the marginal artery. The curved arrow indicates the left anterior descending coronary artery. The white circle overlies the approximate location of the base of the anterior papillary muscle of the left ventricle.

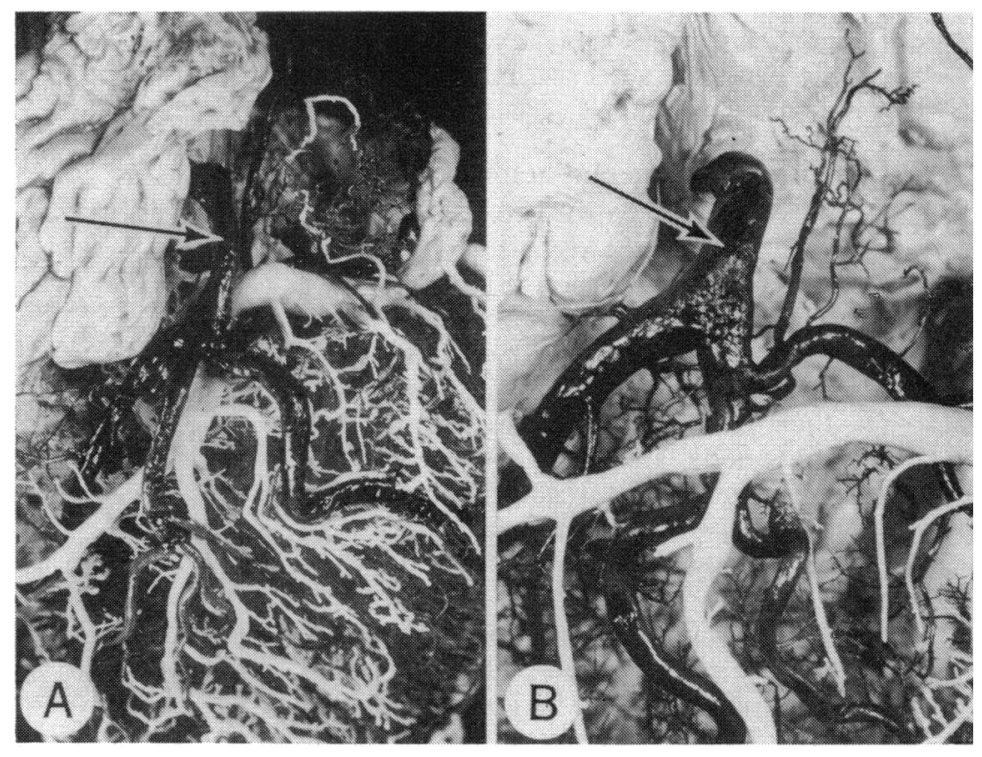

FIG. I In these two vinylite casts of human

heart the main left coronary artery (arrow)

and the complex nature of its primary divisions

are demonstrated. In A, two diagonal branches

are of a calibre the size of the left anterior

descending and circumflex rami; in $\mathrm{B}$, there is

one large and one small diagonal branch, but

the former divides immediately into two major

trunks. These photographs and all others of

vinylite casts are adapted from originals pub-

lished in Anatomy of the Coronary Arteries

(Fames, 1961 ) and are reproduced with the

permission of Paul B. Hoeber, the publisher.

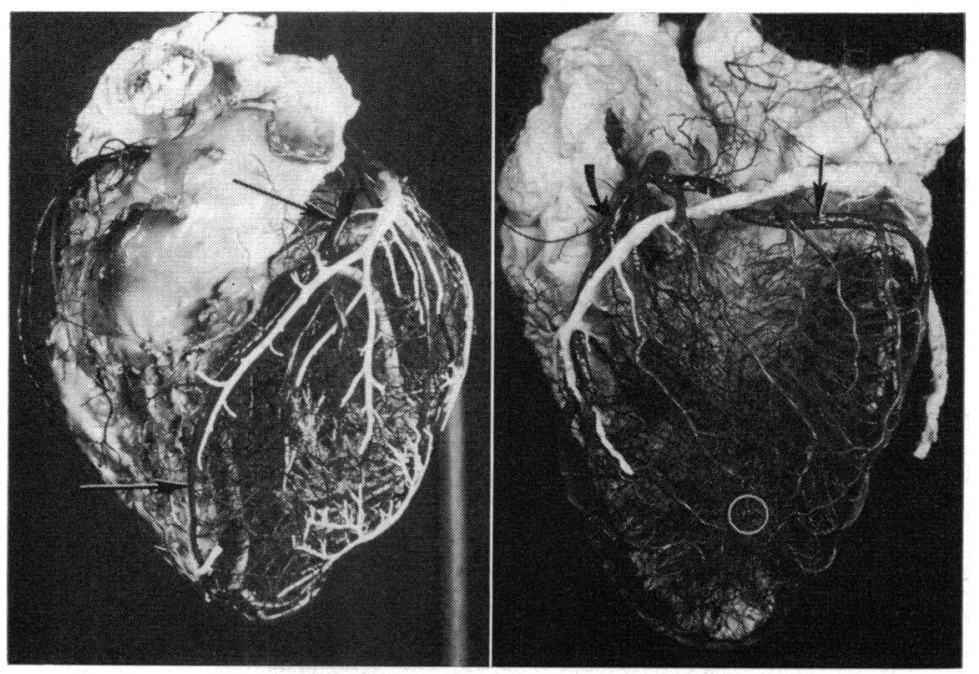

FI G 2

FIG. 3 
subendocardial region of the left ventricle, but significant connexions may be present between any two adjacent major coronary trunks (James, 1970).

\section{Blood supply of special regions}

The sinus node in man is supplied by a proximal branch of the right coronary artery in about 55 per cent of hearts (Fig. 7), and by a proximal branch of the left circumflex in the other 45 per cent. The primary supply is virtually always by a single branch and origins other than those indicated are exceptional. The sinus node artery is consistently the largest atrial artery and in addition to supplying the node also furnishes a major portion of the blood supply to each atrial wall and their septum.

The AV node in man is supplied by a special branch of the right coronary artery in about 90 per cent of hearts, and by a similar branch of the left circumflex artery in the remaining Io per cent. The AV node artery originates from the apex of the penetrating U-turn made by its parent vessel directly beneath the posterior interventricular vein near its junction with the coronary sinus (Fig. 8). From this origin it penetrates the posterior margin of the AV node, passing through for a variable distance into the His bundle. This artery terminates with a characteristic right

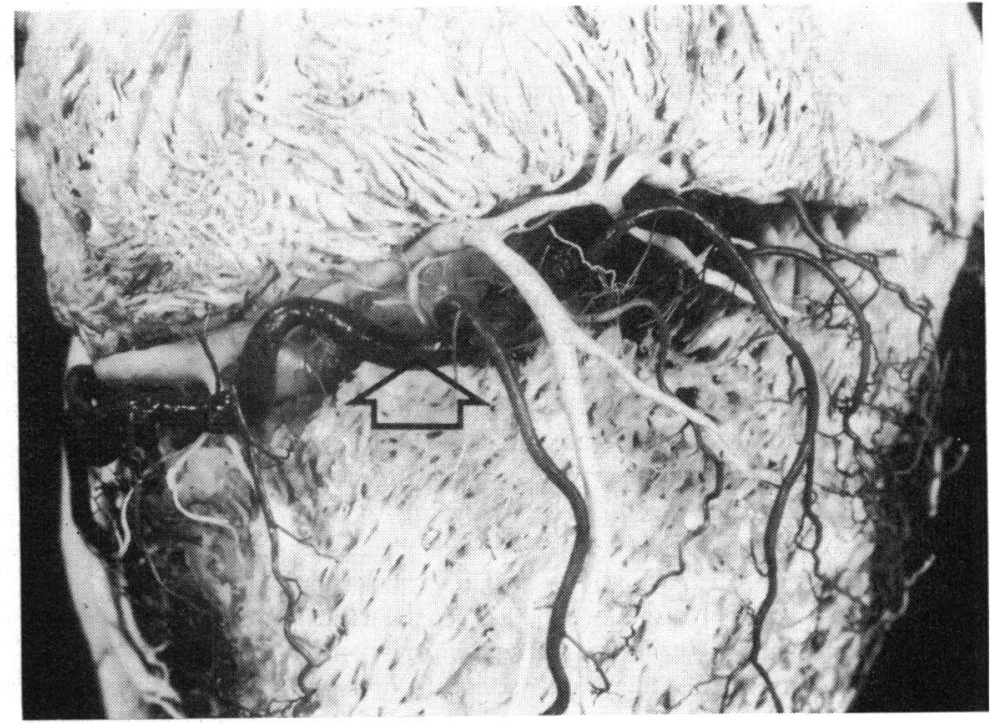

FIG. 4 The right coronary artery (arrow) is shown here lying deep within the right atrioventricular sulcus. The anterior cardiac veins are seen crossing the artery to drain into the right atrium.

angle turn down into the interventricular septum (Fig. 9). Both the AV node artery and the U-turn are readily visualized in good

FIG. 5 The blood supply of the diaphragmatic surface of the human heart is illustrated here with $a$ cast and an orienting drawing. $R A$ is right atrium, $L A$ left atrium, $R V$ right ventricle, $C S$ coronary sinus, and $A V N$ the AV node. The white circle on the cast indicates the approximate location of the base of the posterior papillary muscle of the left ventricle.
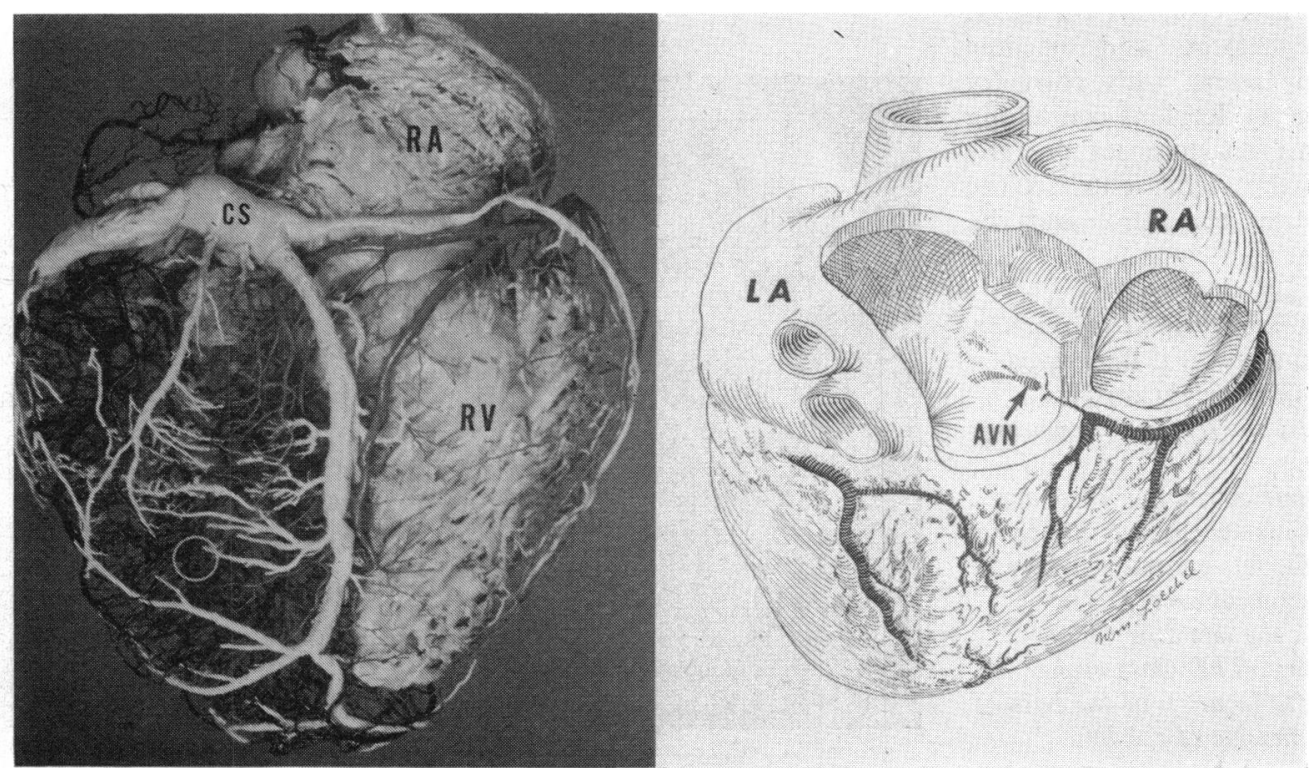

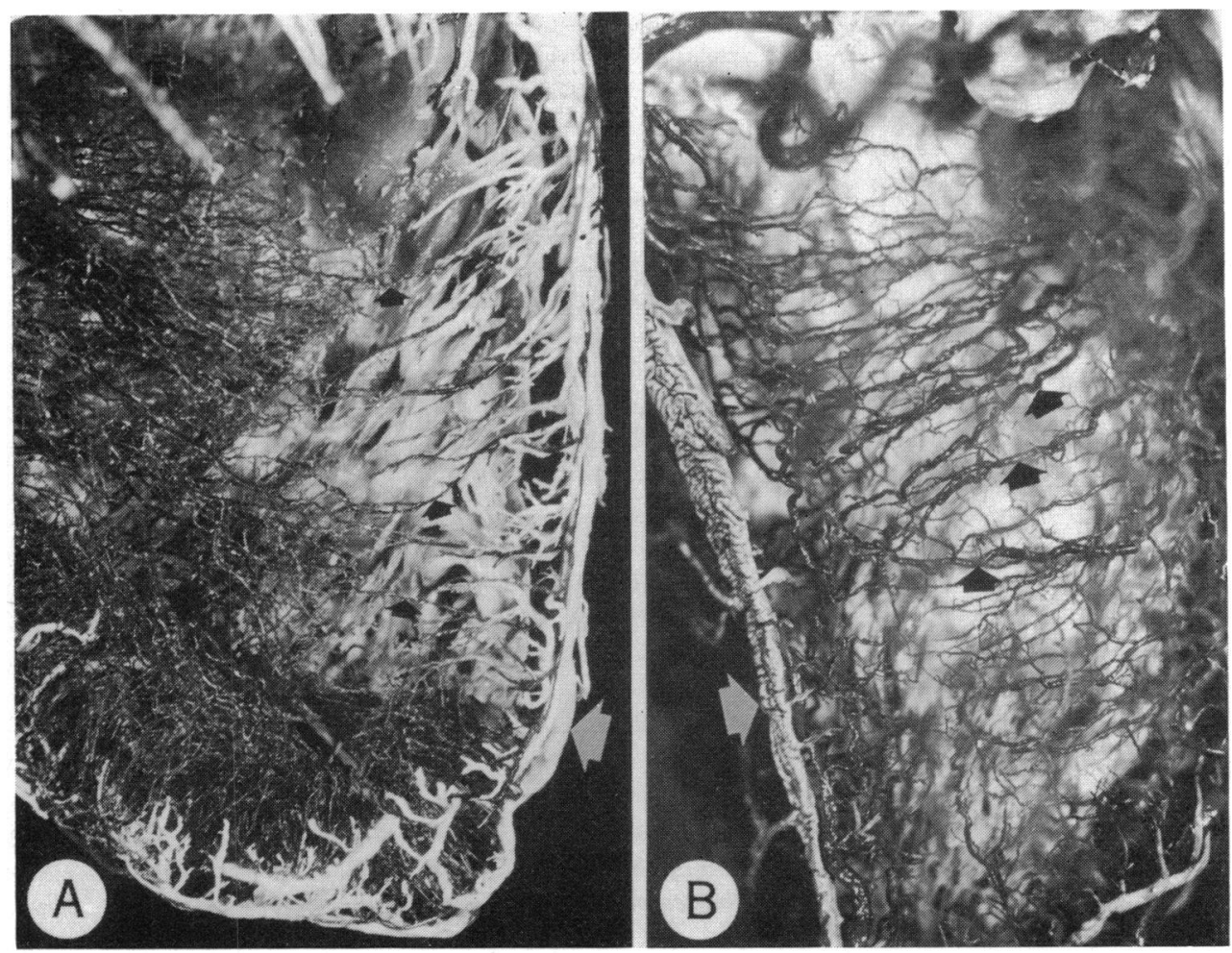

FIG. 6 Intercoronary anastomoses in a normal human heart are shown coursing through the interventricular septum in A. The enlargement of similar anastomoses in an atherosclerotic heart are shown in $\mathrm{B}$. The three small black arrows in each picture point to only a few of the anastomoses, and the white arrow in each indicates the posterior interventricular vein.

The anastomoses in $\mathrm{A}$ are several hundred microns in minimal diameter, and are viewed over the uncast left ventricle. Those in B are up to I $\mathrm{mm}$. in diameter and are viewed over the uncast right ventricle, with the foreground arterial branches intentionally out of focus. Each cast is slightly magnified photographically by approximately the same amount.

quality arteriograms and form useful anatomical landmarks (James, 1962).

The distal His bundle and its branches are supplied variably by the AV node artery and Kugel's artery (arteria anastomotica auricularis magna) (Kugel, 1928), but also by septal branches of the left anterior descending artery.

The papillary muscles of the left ventricle are supplied by those main arteries coursing over their base. For the anterior papillary muscle this includes branches of the left anterior descending and circumflex arteries, and for the posterior papillary muscle not only these two major trunks but also terminal branches of the right coronary artery (Fig. 3 and 5). The important point is that the primary supply to the papillary muscles is virtually always derived from more than one major coronary artery.
The interventricular septum in the human heart is supplied predominantly by septal branches of the left anterior descending coronary artery (Fig. IO). Smaller branches penetrating its posterior margin originate from the posterior descending artery and are an important source for transseptal anastomotic circulation when the anterior descending artery is occluded. Since the anterior descending artery virtually encircles the apex cordis, the apical third of the interventricular septum receives all its blood supply from this vessel.

\section{Clinicopathologic correlations}

Atrial arrhythmias which begin during the course of an acute myocardial infarction are regularly associated with ischaemic injury of the sinus node due to a coronary occlusion proximal to the origin of the sinus node artery 

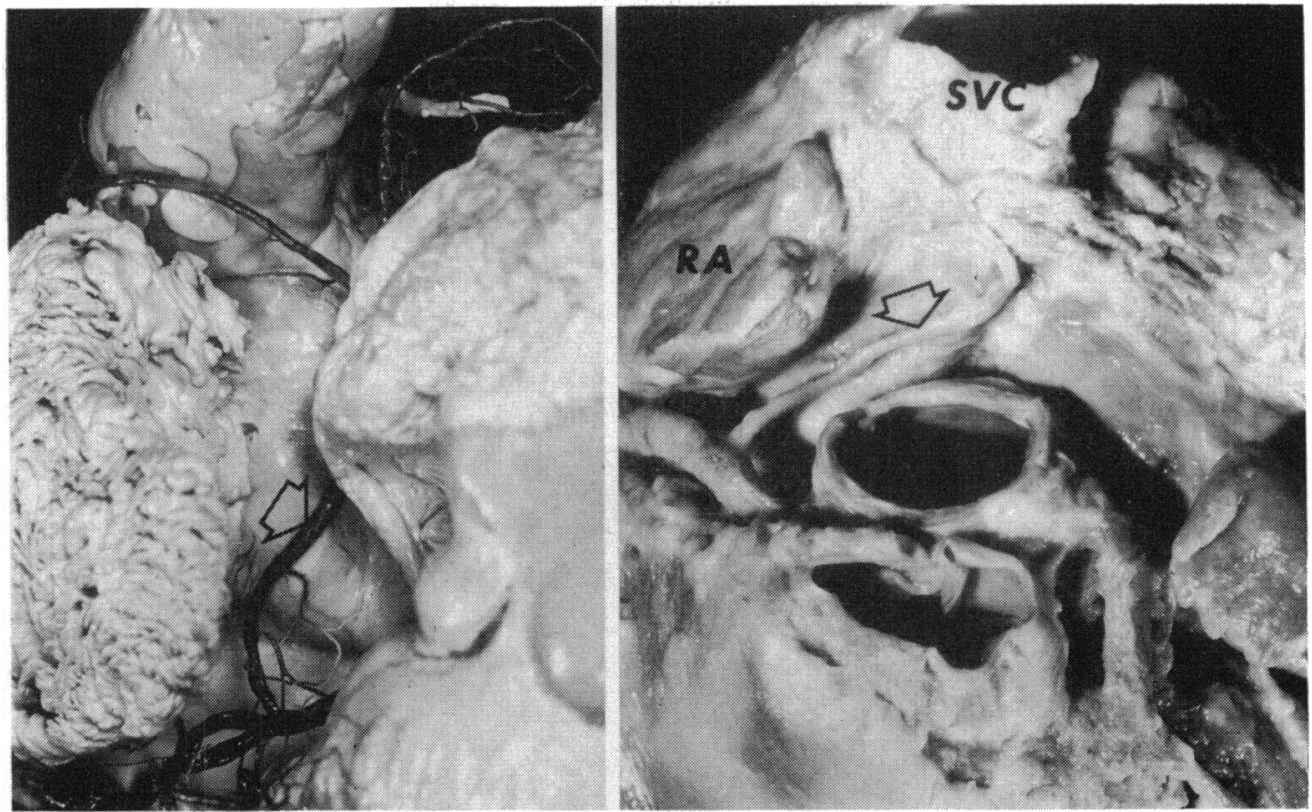

FIG. 7 The sinus node artery (open arrow) is shown here in a cast and in a dissection from two human hearts. In both it originates from the proximal portion of the right coronary artery and ascends along the wall of the right atrium $(R A)$ to the region of the superior vena cava $(S V C)$.

(James, 1968). This clinical event is thus a localizing factor useful in predicting the site of an acute coronary occlusion. For example, an acute posterior myocardial infarction associated with the onset of atrial fibrillation is nearly always due to a proximal right coronary occlusion.

$\mathrm{AV}$ block is a frequent feature of acute posterior myocardial infarction because the region infarcted usually includes the AV node (James, 1968). Such patients often give a history of syncopal attacks before the infarction, and their syncope is sometimes concomitant with anginal pain. Although AV block in clinically observed acute posterior infarction is usually transient and rarely continues as a permanent problem, this is often misinterpreted as a benign phenomenon. No form of unstable AV conduction is safe, and such patients must be observed carefully. The number of subjects with sudden AV block and a lethal arrhythmia beginning during acute infarction, but who never reach the hospital or doctor's office, is unknown. AV block beginning during the course of an acute anteroseptal infarction is known to be associated with a high mortality. The reason for this is apparent from a consideration of the blood supply to the human AV node and
His bundle: for these structures to suffer irreversible ischaemic damage, it is usually necessary for both the right coronary and the left anterior descending branch to be occluded (or for comparable multiple vessel occlusions to be present in the variations of arterial anatomy). Isolated anterior descending artery occlusion may on occasion be associated with bundle-branch block, but it cannot explain sustained complete AV block.

Rupture of the interventricular septum may be considered in the same fashion as the pathogenesis of sustained AV block, which often precedes or accompanies it. Both the anterior and posterior sources of blood supply must be occluded for the septum to be sufficiently deprived of potential collateral circulation so that it can perforate. This means an occlusion of the proximal portion of the left anterior descending coronary artery plus an occlusion of the right coronary artery, one usually having preceded the other by some period of time. The patient with an old posterior infarct who subsequently develops an acute anteroseptal infarct is a candidate both for complete AV block and for septal rupture, and must be observed carefully for these complications.

Papillary muscle dysfunction during acute 
FIG. 8 The U-turn of the right coronary artery (open arrow) is shown here in a vinylite cast viewed from directly above, and in a coronary arteriogram viewed in the left anterior oblique projection. The left atrium is cast white but the right atrium is uncast. In the arteriogram (performed by Dr. Sven Paulin and reproduced with his permission) the lower black arrow points to the $A V$ node artery originating from the apex of the $U$ turn; the upper black arrow points to the sinus node artery and the white arrow to the conus artery. The dotted line from the U-turn to the apex of the heart separates the right and left ventricles on the diaphragmatic surface of the heart. The number 2 refers to sequence in the series of exposures.

myocardial infarction leads to decreased efficiency of left ventricular ejection, and papillary muscle rupture is often fatal owing to massive mitral valvular regurgitation. Ischaemic injury of the papillary muscles rarely occurs without more than one major coronary being occluded, since the pertinent blood supply is virtually always of multiple origin; but, just as with septal rupture, the responsible lesions rarely occur at the same time, some occlusions having been present a variable length of time before the finally responsible one.

\section{Epilogue}

In this brief overview many important advances in our understanding of the coronary circulation in myocardial infarction are necessarily omitted. For the sake of clarity much of the discussion dealt with lesions in single coronary arteries and their consequences, although it must be recognized that in the patient multiple lesions are the rule rather than the exception. Similarly, only a few clinicopathologic correlations were presented, to illustrate their usefulness in understanding the pathophysiology of myocardial infarction. It is an exciting time for those interested in the study and optimal treatment of acute

FIG. 9 This close-up photograph illustrates the arterial supply of the region of $A V$ node and His bundle in the human heart, viewed across the uncast left ventricle. The $A V$ node artery (black arrow on the right) terminates in its characteristic right angle branch, which occurs approximately in the midpoint of the $A V$ node (not seen); other arterial branches to the region come from the first major septal artery (black arrow on the left). The void in the cast represents the location of the crista supraventricularis (CS).
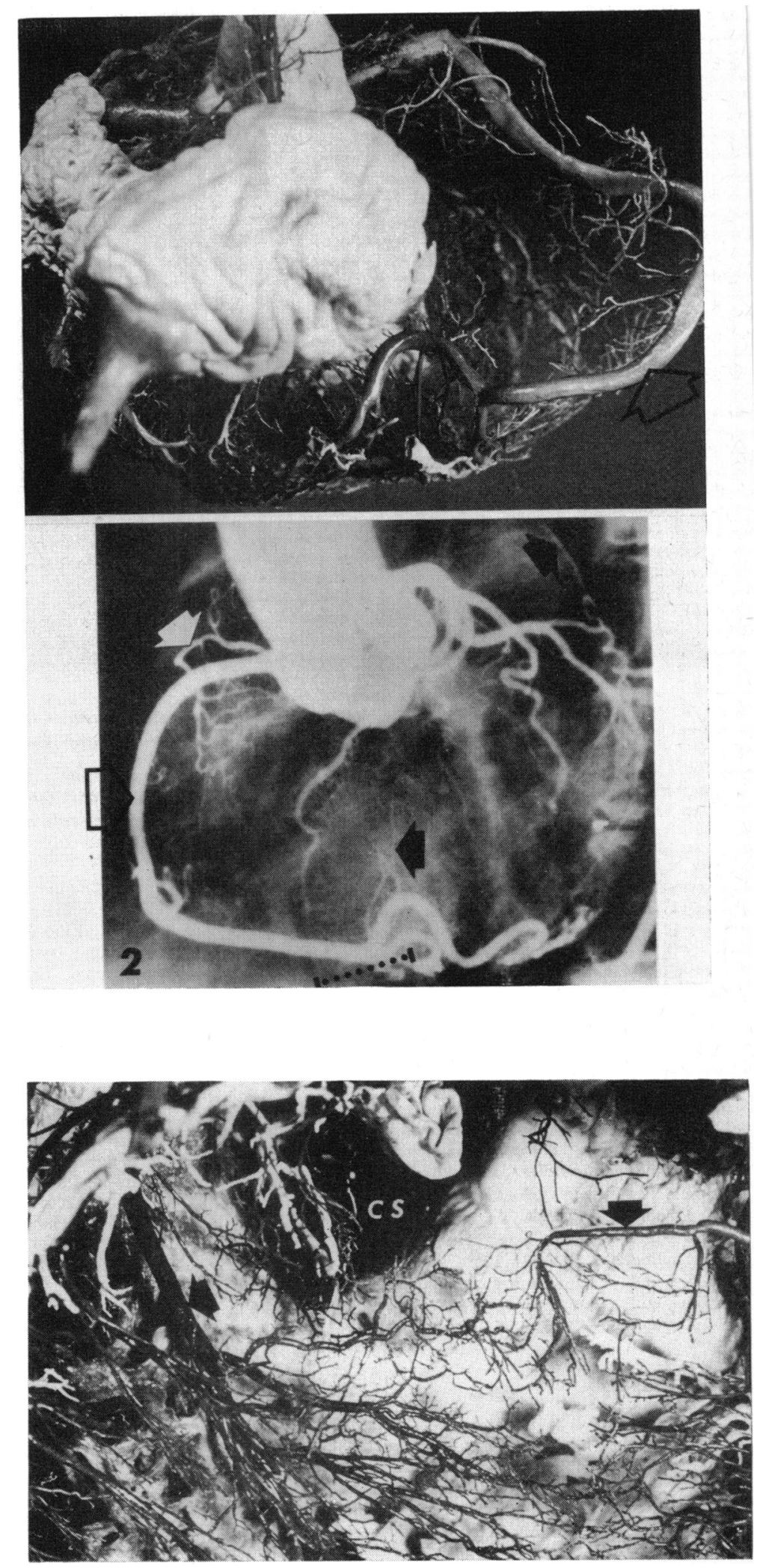

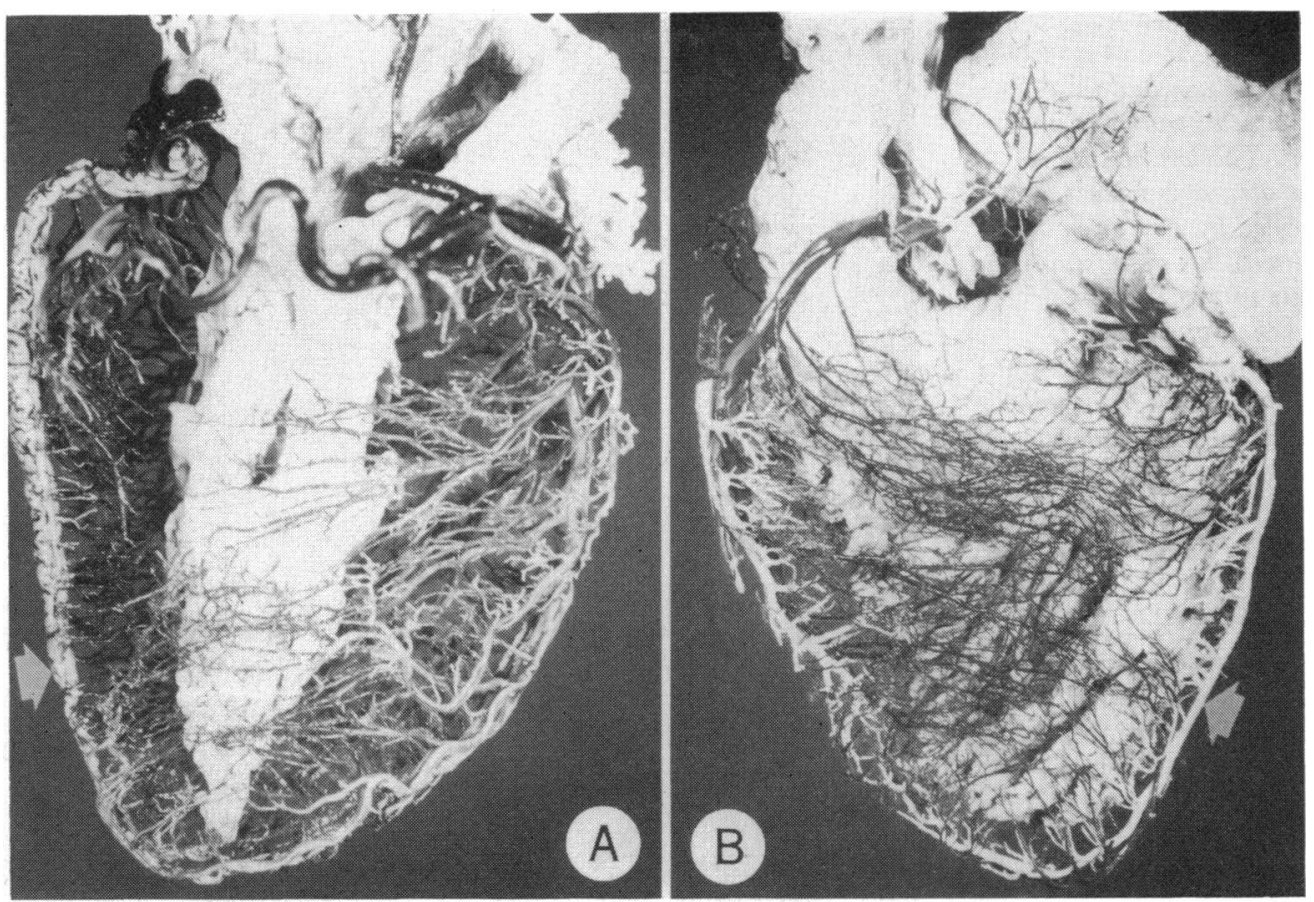

FIG. Io The blood supply of the human interventricular septum is illustrated with casts of two different hearts. A is viewed across the uncast right ventricle, and B over the uncast left ventricle. In both, the arteries of the free ventricular wall have been cut away to simplify seeing the septum. A white arrow indicates the posterior interventricular vein. Note that the apical third of the septum is supplied entirely by branches of the left anterior descending artery.

myocardial infarction, and with the current wide utilization of increasingly sophisticated methods and techniques of research it may be anticipated that new advances are continually imminent.

\section{References}

Eliớ, R. S., and Bratt, G. (1969). The paradox of myocardial ischemia and necrosis in young women with normal coronary arteriograms. American Fournal of Cardiology, 23, 633.

James, T. N. (196I). Anatomy of the Coronary Arteries. Hoeber, New York.

- (1962). A useful landmark for interpreting angiocardiograms. Radiology, 79, 804.

- (1968). The coronary circulation and conduction system in acute myocardial infarction. Progress in Cardiovascular Disease, ro, 410. 